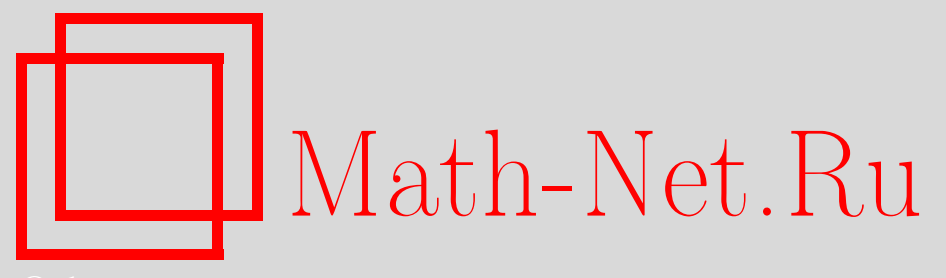

Е. Смирнов, О квадратичных вычетах, Квант, 2019, номер 10, 2-11

DOI: https://doi.org/10.4213/kvant20191001

Использование Общероссийского математического портала Math-Net.Ru подразумевает, что вы прочитали и согласны с пользовательским соглашением http://www.mathnet.ru/rus/agreement

Параметры загрузки:

IP : 54.147 .182 .235

26 апреля 2023 г., $12: 33: 57$

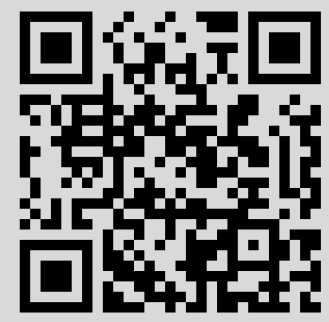




\section{квадратичных вычетах}

\section{E. СМИРНОВ}

$\mathrm{M}$ НОГИЕ ЗНАЮТ, ЧТО С ОСТАТКАМИ при делении на данное натуральное число $m$ - также говорят «с остатками по модулю $m$ » и пишут «mod $m$ » - можно делать почти то же самое, что с целыми числами: остатки можно складывать, вычитать, умножать... А если $m$ равно простому числу $p$, то остатки можно даже и делить! Так, каждое линейное уравнение $a x=b(\bmod p)$ имеет единственное решение, если $a-$ ненулевой остаток.

А как обстоит дело с квадратными уравнениями? Как выяснить, есть ли решения у уравнения $a x^{2}=b$ по модулю простого числа $p$ ? Этому и посвящена настоящая статья. Главным утверждением, которое мы при этом докажем, будет знаменитый квадратичный закон взаимности Гаусса - теорема, устанавливающая связь между разрешимостью уравнений $x^{2}=p(\bmod q)$ и $x^{2}=q(\bmod p)$ для двух разных простых чисел $p$ и $q$. С его помощью мы научимся выяснять, разрешимо ли квадратное уравнение по данному простому модулю.

\section{Арифметика остатков}

В этом разделе мы кратко напомним некоторые факты об арифметике вычетов по простому модулю. Читателю, который не знаком с арифметикой остатков, рекомендуем сначала прочесть, например, статьи: Н.Виленкин. Сравнения и классы вычетов («Квант» №10 за 1978 г.) или A.Егоров, А.Котова. Необыкновенные арифметики («Квант» №3-4 за 1993 г.).

Чтобы сложить два остатка $a$ и $b$ по модулю $m$, нужно сложить их как целые числа и взять у результата остаток по модулю $m$. Так же определяются и разность, и произведение остатков. Определенные таким образом операции удовлетворяют обычным законам арифметичес-

DOI: https://doi.org/10.4213/kvant20191001 ких действий: для них верны переместительный, сочетательный и распределительный законы, существуют ноль и единица. (Алгебраисты сказали бы, что они образуют кольцо.) Подробности читатель может восстановить сам или посмотреть в одной из упомянутых выше статей.

Для примера составим таблицы умножения остатков по модулю 6 и 7. При этом мы опускаем строчку и столбец, отвечающие

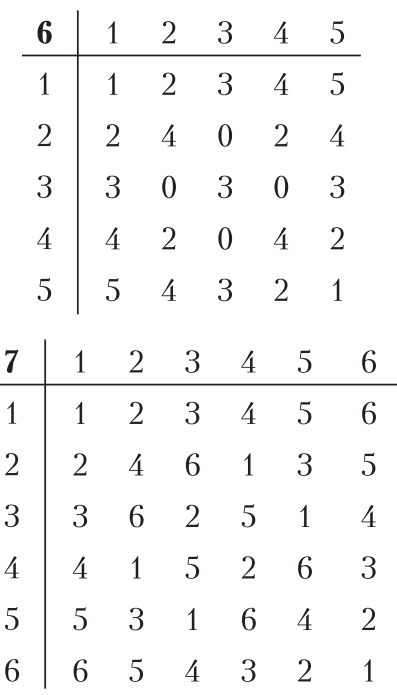

умножению на ноль: они состоят из одних нулей.

Сопоставив эти таблицы, можно сделать некоторые наблюдения. Так, например, в первой из таблиц (по модулю 6) встречаются нули; иначе говоря, существуют такие два ненулевых остатка, произведение которых равно нулю. Такие остатки называются делителями нуля. Скажем, по модулю 6 делителями нуля будут числа 2, 3 и 4.

Ясно, что по составному модулю делители нуля всегда будут: так, если $m$ составное, то существуют такие $k$ и $l$, отличные от единицы, для которых $m=k l$. Поэтому числа $k$ и $l$ соответствуют ненулевым ос- 
таткам, произведение которых равно нулю по модулю $m$.

Чуть менее тривиален обратный вопрос: верно ли, что в арифметике остатков по простому модулю делителей нуля нет? (Например, по модулю 7 их нет, что подтверждается второй таблицей.) Оказывается, что это тоже верно.

Докажем это. Рассмотрим арифметику остатков по модулю $p$ и предположим противное: пусть нашлись такие $k$ и $l$, которые дают при делении на $p$ ненулевые остатки - но при этом их произведение делится на $p$ нацело, т.е. $k l=p m$. Однако если произведение двух чисел делится на простое число $p$, то на $p$ делится хотя бы одно из этих чисел. Противоречие: ни $k$, ни $l$ не делятся на $p$.

Мы получили следующее утверждение.

Предложение 1. В арифметике остатков по модулю $m$ нет делителей нуля тогда и только тогда, когда т является простым числом.

Далее нас будет интересовать в основном тот случай, когда $m$ простое; в этом случае будем обозначать его буквой $p$, а соответствующее множество остатков (с операциями сложения и умножения) будет обозначаться через $\mathbb{Z} / p \mathbb{Z}$. $^{1}$

Можно сделать еще одно наблюдение: в каждой из строк второй таблицы записаны в каком-то порядке все ненулевые остатки по модулю 7; все числа от 1 до 6 встречаются в ней по одному разу, и ни одно не повторяется. Оказывается, что так обстоит дело и для произвольного простого модуля.

Предложение 2. Пусть $p$ простое, $a \in \mathbb{Z} / p \mathbb{Z}$ - некоторый ненулевой остаток. Тогда среди остатков $0, a, 2 a, 3 a, \ldots$ ..., $(p-1)$ а нет повторяюшихся.

Доказательство. Пусть два остатка $k, m \in \mathbb{Z} / p \mathbb{Z}$ таковы, что $a k=a m$. Тогда $a(k-m)$ равно нулю. Но мы только что

1 Смысл этого обозначения в том, что мы рассматриваем целые числа, множество которых традиционно обозначается через $\mathbb{Z}$, с точностью до прибавления чисел, делящихся на $p$, множество которых естественно обозначить через $p \mathbb{Z}$. доказали, что в $\mathbb{Z} / p \mathbb{Z}$ произведение двух ненулевых остатков отлично от нуля. Поскольку по условию $a \neq 0$, то это значит, что $k-m=0$, т.е. $k=m$, что и требовалось.

Из этого несложного предложения вытекает несколько интересных следствий.

Следствие 1. $B \mathbb{Z} / p \mathbb{Z}$ линейное уравнение ах $=0$ при $a \neq 0$ имеет единственное решение.

Доказательство. Действительно, переберем все возможные значения $x$, т.е. рассмотрим все остатки вида $0, a, 2 a, \ldots$ Согласно предложению 2 , среди них будет остаток, равный $b$, причем ровно один.

Частным случаем этого следствия является следующее утверждение.

Следствие 2. Для каждого ненулевого остатка $a \in \mathbb{Z} / p \mathbb{Z}$ существует и единственный обратный, т.е. такой остаток $x$, что $a x=1$.

Этот остаток обычно обозначается $a^{-1}$.

Пример 1. Например, в $\mathbb{Z} / 7 \mathbb{Z}$ обратным к остатку 3 будет остаток 5: действительно, $3 \cdot 5=15$, что дает остаток 1 по модулю 7.

Замечание. Отметим, что в арифметике по составному модулю ни то, ни другое следствие не будет выполняться. Так, например, по модулю 6 уравнение $2 x=4$ будет иметь два решения: 2 и 5, а ни один из остатков 2, 3 и 4 не будет обратим.

Упражнение 1. Выясните, какие остатки будут обратимы в арифметике остатков по произвольному составному модулю $m$.

А что будет, если перемножить все остатки $a, 2 a, \ldots,(p-1) a$ ? С одной стороны, $a^{p-1} \cdot 1 \cdot 2 \cdot \ldots \cdot(p-1)=a^{p-1}(p-1) !$. С другой стороны, согласно предложению 2 , эти остатки - это те же $1,2, \ldots, p-1$, только записанные в другом порядке. Значит, их произведение равняется $(p-1)$ !. Тем самым мы получаем равенство

$$
a^{p-1}(p-1) !=(p-1) ! .
$$

Поделив это равенство на $(p-1)$ ! (контрольный вопрос: почему это можно сделать?), получаем следующую теорему.

Теорема 1 (малая теорема Ферма). Пусть $p$ простое. Тогда в $\mathbb{Z} / p \mathbb{Z}$ для 
любого ненулевого а верно равенство

$$
a^{p-1}=1 .
$$

Упражнение 2. Покажите, что остатки, обратные к самим себе, т.е. такие, для которых $a=a^{-1}$, это в точности 1 и $p-1$. Выведите отсюда теорему Вильсона:

$$
(p-1) !=-1 \text {. }
$$

\section{Квадратичные вычеты}

Первая в истории человечества книга по алгебре, «Китаб аль-джебр ва-ль-мукабала», была написана средневековым ученым Мухаммадом ибн Мусой аль-Хорезми около 830 года н.э. Аль-Хорезми был уроженцем Хивы - сейчас это территория Узбекистана. В этой книге, в числе прочего, был изложен метод решения квадратного уравнения.

Основной метод решения квадратного уравнения (с вещественными коэффициентами) состоит в выделении полного квадрата, что сводит его к уравнению вида $x^{2}=a$. Такое уравнение легко решить: если $a>0$, то его корни равны $\pm \sqrt{a}$; если $a=0$, то единственный корень равен нулю; если же $a<0$, то корней у такого уравнения нет.

Квадратичные вычеты и невычеты. Наша ближайшая задача будет состоять в том, чтобы научиться искать число решений уравнения $x^{2}=a$, где $a \in \mathbb{Z} / p \mathbb{Z}$ остаток по некоторому фиксированному простому модулю $p$. Будем считать, что $p>2$, т.е. $p$ - нечетное простое число.

Заметим, что если уравнение $x^{2}=a$ при $a \neq 0$ имеет корень, то этих корней обязательно будет ровно два: а именно, если $b-$ корень этого уравнения, то и $-b-$ тоже его корень. При этом, поскольку $p$ нечетно, $b \neq-b$.

Поэтому получается, что $x^{2}-a=$ $=(x-b)(x+b)$. Отсюда следует, что других корней у уравнения $x^{2}=a$ нет: если $c$ - корень уравнения, то $(c-b)(c+b)=0$, откуда $c=b$ или $c=-b$ (обратите внимание, что здесь мы опять пользуемся отсутствием делителей нуля в $\mathbb{Z} / p \mathbb{Z}$ !).

Определение 1. Пусть $a \in \mathbb{Z} / p \mathbb{Z}, a \neq 0$. Будем называть а квадратичным выче- том, если уравнение $x^{2}=a$ разрешимо, и квадратичным невычетом в противном случае.

Пример 2. По модулю 7 квадратичными вычетами будут числа 1, 2 и 4: это в точности те числа, которые стоят на диагонали в таблице умножения остатков по модулю 7. Отметим, что каждое из них встречается там ровно два раза.

При этом число 0 мы не будем относить ни к квадратичным вычетам, ни к невычетам (так же, как вещественное число 0 не является ни положительным, ни отрицательным).

Предложение 3. Количество как квадратичных вычетов, так и квадратичных невычетов в $\mathbb{Z} / p \mathbb{Z}$ равно $\frac{p-1}{2}$.

Доказательство. Каждому квадратичному вычету $a$ соответствуют два решения $\pm b$ уравнения $x^{2}=a$. Также ясно, что каждый ненулевой остаток из $\mathbb{Z} / p \mathbb{Z}$ является решением ровно одного квадратного уравнения такого вида. Поэтому получается, что квадратичных вычетов оказывается вдвое меньше, чем ненулевых элементов из $\mathbb{Z} / p \mathbb{Z}$, т.е. $\frac{p-1}{2}$. Все остальные элементы $\mathbb{Z} / p \mathbb{Z}$ тогда будут квадратичными невычетами, и их $(p-1)-\frac{p-1}{2}$, а значит, столько же.

\section{Упражнения}

3. Для всех нечетных простых чисел $p<20$ перечислите все квадратичные вычеты и невычеты по модулю $p$.

4. Докажите, что при нечетном простом $p$ квадратное уравнение $a x^{2}+b x+c=0$, где $a, b, c \in \mathbb{Z} / p \mathbb{Z}, a \neq 0$,

- имеет два решения, равные $x_{1,2}=\frac{b \pm \sqrt{D}}{2 a}$, если его дискриминант $D=b^{2}-4 a c$ является квадратичным вычетом;

- имеет единственное решение, если $D=0$;

• неразрешимо над $\mathbb{Z} / p \mathbb{Z}$, если $D$ - квадратичный невычет.

Указание. С помощью выделения полного квадрата сведите данное уравнение к виду $x^{2}=D$.

Символ Лежандра и его мультипликативность. Ясно, что произведение двух 
квадратичных вычетов снова будет квадратичным вычетом. А что будет, если перемножить два квадратичных невычета? А вычет и невычет? Оказывается, верна следующая теорема.

Теорема 2. а) Произведение двух квадратичных вычетов - квадратичный вычет;

б) произведение квадратичного вычета и квадратичного невычета - квадратичный невычет;

в) произведение двух квадратичных невычетов - квадратичный вычет.

Доказательство. Пункт а) очевиден: если $a$ и $b$ квадратичные вычеты, т.е. существуют такие $x$ и $y$, для которых $x^{2}=a$ и $y^{2}=b$, то $a b$ тоже будет квадратичным вычетом, поскольку $(x y)^{2}=a b$.

Докажем пункт б). Пусть $a$ - квадратичный вычет, $b$ - квадратичный невычет. Предположим противное: что $a b$ - квадратичный вычет. Поскольку $a-$ квадратичный вычет, найдется такое $x$, что $x^{2}=a$. В таком случае и элемент $a^{-1}$ будет квадратичным вычетом, так как $a^{-1}=\left(x^{-1}\right)^{2}$. Соответственно, в силу пункта а) элемент $b$ как произведение квадратичных вычетов $a b$ и $a^{-1}$ будет квадратичным вычетом - противоречие.

Пункт в) аналогичными формальными манипуляциями доказать уже не удается. Однако его можно вывести из пункта б) при помощи подсчета числа элементов. А именно, пусть $a-$ фиксированный квадратичный невычет. Рассмотрим все элементы вида $a, 2 a, \ldots,(p-1) a$. Как обсуждалось ранее, в этой последовательности все элементы $1,2, \ldots,(p-1)$ встречаются, причем ровно по одному разу - поэтому среди них $\frac{p-1}{2}$ квадратичных вычетов и $\frac{p-1}{2}$ квадратичных невычетов. Однако в силу пункта б) при умножении $a$ на квадратичный вычет получается квадратичный невычет, и таких элементов будет $\frac{p-1}{2}$, т.е. все квадратичные невычеты будут получаться таким образом. Значит, при умножении $a$ на все остальные элементы (т.е. невычеты) должны получаться квадратичные вычеты, что и требовалось.

Тем самым квадратичные вычеты и невычеты ведут себя при умножении примерно так же, как положительные и отрицательные числа. Это мотивирует следующее определение.

Определение 2. Пусть $a \in \mathbb{Z} / p \mathbb{Z}, a \neq 0$. димвол Лежандра $\left(\frac{a}{p}\right)$ определяется сле$\left(\frac{a}{p}\right)= \begin{cases}1, & a-\text { квадратичный вычет; } \\ -1, & a-\text { квадратичный невычет; } \\ 0, & a=0 .\end{cases}$

Таким образом, символ Лежандра можно считать аналогом функции знака числа. Тогда предыдущая теорема принимает следующий компактный вид.

Теорема 3. Символ Лежандра мультипликативен:

$$
\left(\frac{a}{p}\right)\left(\frac{b}{p}\right)=\left(\frac{a b}{p}\right) .
$$

Далее мы приведем другое доказательство этой теоремы, которое будет следовать из еще одного полезного утверждения, позволяющего вычислить символ Лежандра, - критерия Эйлера.

Критерий Эйлера. Прежде чем читать дальше, рекомендуем читателю выполнить следующее упражнение.

Упражнение 5. Возьмите какое-нибудь нечетное простое число, меньшее 20, и возведите каждый из ненулевых остатков $a \in \mathbb{Z} / p \mathbb{Z}$ в степень $\frac{p-1}{2}$. Убедитесь, что у вас всегда будет получаться \pm 1 . А когда получается 1 , а когда -1 ?

Тем самым вы «экспериментально» проверите утверждение следующей теоремы.

Теорема 4 (критерий Эйлера). Пусть $p>2$ - простое число, $a \in \mathbb{Z} / p \mathbb{Z}$. Тогда

$$
a^{\frac{p-1}{2}}=\left(\frac{a}{p}\right) \text {. }
$$

Доказательство. При $a=0$ утверждение теоремы очевидно. Дальше будем считать, что $a$ отлично от 0 .

Во-первых, сначала убедимся, что при 
любом $a \neq 0$ будет иметь место равенство $a^{\frac{p-1}{2}}= \pm 1$. Действительно, $\left(a^{\frac{p-1}{2}}\right)^{2}=$ $=a^{p-1}=1$ по малой теореме Ферма. Но остаток, квадрат которого равен 1, может равняться только 1 или -1 .

$\underset{\underline{p-1}}{\text { Далее, ясно, что если }}\left(\frac{a}{p}\right)=1$, то $a^{\frac{p-1}{2}}=1$. Действительно, если $a-$ квадратичный вычет, то существует такое $b$, что $b^{2}=a$. Тогда $a^{\frac{p-1}{2}}=\left(b^{2}\right)^{\frac{p-1}{2}}=b^{p-1}=1$ опять-таки в силу малой теоремы Ферма.

Осталось доказать, что если $a-$ квадратичный невычет, то $a^{\frac{p-1}{2}}=-1$. Для этого рассмотрим уравнение $x^{\frac{p-1}{2}}-1=0$. Это полиномиальное уравнение степени $\frac{p-1}{2}$, следовательно, по теореме Безу (которая верна и для многочленов с коэффициентами из $\mathbb{Z} / p \mathbb{Z}$ ) у него не может быть более $\frac{p-1}{2}$ корней. Но такое количество корней нам уже известно: это все квадратичные вычеты. Тем самым получается, что квадратичные невычеты не являются корнями этого уравнения, т.е. для них $a^{\frac{p-1}{2}} \neq 1$. Значит, для них $a^{\frac{p-1}{2}}=-1$. Теорема доказана.

Для полноты изложения докажем, что многочлен с коэффициентами из $\mathbb{Z} / p \mathbb{Z}$ степени $d$ имеет не более $d$ корней. Это доказывается точно так же, как и для многочленов с вещественными коэффициентами. Докажем это индукцией по $d$. База при $d=1$ очевидна: это следствие 1. Для доказательства индуктивного перехода отметим, что если $p(x)$ - многочлен степени $d$, коэффициенты которого принадлежат $\mathbb{Z} / p \mathbb{Z}$, а остаток $a \in \mathbb{Z} / p \mathbb{Z}$ является его корнем, т.е. $p(a)=0$, то $p(x)$ делится на двучлен $x-a$, значит, представляется в виде $p(x)=q(x)(x-a)$. Для доказательства этого факта разделим $p(x)$ на $x-a$ с остатком: $p(x)=q(x)(x-a)+r$ и подставим в качестве $x$ значение $a$. Левая часть окажется равной нулю, стало быть, и $r=0$. Степень многочлена $q(x)$ будет на единицу меньше, чем степень $p(x)$, а значит, и корней у него не более $d-1$ по предположению индукции. А корни многочлена $p(x)$ - это $a$ и корни многочлена $q(x)$ (здесь мы используем отсутствие делителей нуля в $\mathbb{Z} / p \mathbb{Z}$ ). Поэтому их не больше $d$, что и требовалось.

Из критерия Эйлера очевидно следует мультипликативность символа Лежандра. Действительно,

$$
\left(\frac{a}{p}\right)\left(\frac{b}{p}\right)=a^{\frac{p-1}{2}} b^{\frac{p-1}{2}}=(a b)^{\frac{p-1}{2}}=\left(\frac{a b}{p}\right) .
$$

Кроме того, с помощью критерия Эйлера легко понять, когда -1 является квадратичным вычетом, а когда - невычетом:

Следствие 3. Имеет место равенство

$$
\left(\frac{-1}{p}\right)=(-1)^{\frac{p-1}{2}}= \begin{cases}1, & p=4 k+1 \\ -1, & p=4 k+3 .\end{cases}
$$

«Положительные» и «отрицательные» остатки. Выпишем все элементы из $\mathbb{Z} / p \mathbb{Z}$ в следующем порядке:

$$
-\frac{p-1}{2}, \ldots,-2,-1,0,1,2, \ldots, \frac{p-1}{2} .
$$

Условимся называть те из них, которые выписаны слева от нуля, «отрицательными», а те, что справа - «положительными». ${ }^{2}$

Рассмотрим произвольный ненулевой элемент $a \in \mathbb{Z} / p \mathbb{Z}$ и умножим его на все «положительные» элементы. Получим элементы $a, 2 a, \ldots, \frac{p-1}{2} a$.

Нетрудно доказать следующее утверждение.

Предложение 4. Множество $\left\{a, 2 a, \ldots, \frac{p-1}{2} a\right\}$ содержит ровно по одному элементу из каждой пары $\pm 1, \pm 2, \ldots, \pm \frac{p-1}{2}$.

Доказательство. Ранее мы уже видели, что разные элементы из $\mathbb{Z} / p \mathbb{Z}$ при умно-

\footnotetext{
${ }^{2}$ Не надо путать положительные/ отрицательные элементы в смысле этого обозначения с квадратичными вычетами/невычетами.
} 
жении на произвольное ненулевое $a$ не могут переходить в один и тот же элемент: это противоречило бы тому, что в $\mathbb{Z} / p \mathbb{Z}$ нет делителей нуля.

Докажем, что для различных «положительных» элементов $k \neq l$ элементы $a k$ и al не могут быть противоположны друг другу. Действительно, это значило бы, что

$$
0=a k+a l=a(k+l),
$$

откуда $k+l=0(\bmod p)$. Но этого не может быть: поскольку $0<k, l \leq \frac{p-1}{2}$, то $0<k+l<p$, значит, $k+l \neq 0(\bmod p)$.

Поэтому среди элементов $a, 2 a, \ldots, \frac{p-1}{2} a$ встречается не более одного элемента из каждой пары $\pm k$. Но элементов и пар по $(p-1) / 2-$ стало быть, из каждой пары $\pm k$ войдет ровно один остаток.

Обозначим через $\varepsilon(a)$ количество «отрицательных» элементов во множестве $\left\{a, 2 a, \ldots, \frac{p-1}{2} a\right\}$, т.е. число таких «положительных» элементов, которые при умножении на $a$ становятся «отрицательными» .

Предложение 5 (лемма Гаусса). Имеет место равенство $(-1)^{\varepsilon(a)}=\left(\frac{a}{p}\right)$. Иначе говоря, $\varepsilon(a)$ четно, если а-квадратичный вычет, и нечетно в противном случае.

Доказательство. Перемножим все остатки $a, 2 a, \ldots, \frac{p-1}{2} a$. В силу предыдущего рассуждения мы получим, что это произведение равно

$$
a \cdot 2 a \cdot \ldots \cdot \frac{p-1}{2} a=(-1)^{\varepsilon(a)} \cdot 1 \cdot 2 \cdot \ldots \cdot \frac{p-1}{2} .
$$

Но, с другой стороны, оно же равняется

$$
\begin{aligned}
a \cdot 2 a \cdot \ldots \cdot \frac{p-1}{2} a=a^{\frac{p-1}{2}} & \cdot 1 \cdot 2 \cdot \ldots \cdot \frac{p-1}{2}= \\
& =\left(\frac{a}{p}\right) \cdot 1 \cdot 2 \cdot \ldots \cdot \frac{p-1}{2}
\end{aligned}
$$

(в последнем равенстве использован критерий Эйлера).

Поделив обе части на отличную от нуля величину $((p-1) / 2)$ ! , получаем требуемое равенство.
Это предложение позволяет вычислить символ Лежандра для некоторых значений $a$.

Пример 3. Убедимся еще раз, что $\left(\frac{-1}{p}\right)=(-1)^{\frac{p-1}{2}}$. Действительно, при умножении на -1 все «положительные» числа становятся «отрицательными» - а их всего $(p-1) / 2$.

Выясним, когда число 2 является квадратичным вычетом по модулю $p$. Оказывается, что это зависит от остатка от деления $p$ на 8: если он равен 1 или 7, то 2 будет квадратичным вычетом, а если 3 или 5, то невычетом.

Предложение 6. Имеет место равенство

$$
\left(\frac{2}{p}\right)=(-1)^{\frac{p^{2}-1}{8}}= \begin{cases}1, & p=8 k \pm 1 \\ -1, & p=8 k \pm 3 .\end{cases}
$$

Доказательство. Умножим все «положительные» элементы на 2; получим элементы вида

$$
2,4, \ldots, p-3, p-1 .
$$

«Отрицательными» будут те из них, которые будут заключены между $p / 2$ и $p-1$. Их количество, как нетрудно видеть, будет равно $\left\lceil\frac{p-1}{4}\right\rceil$ (т.е. числу $\frac{p-1}{4}$, округленному до ближайшего целого вверx).

Эта величина и будет равна $\varepsilon(2)$; мы хотим найти ее четность. Проще всего сделать это, разобрав четыре возможных случая:

• если $p=8 k+1$, то $\varepsilon(2)=\left\lceil\frac{p-1}{4}\right\rceil=2 k$;

- если $p=8 k+3$, то $\varepsilon(2)=\left\lceil 2 k+\frac{1}{2}\right\rceil=$ $=2 k+1$;

- если $p=8 k+5$, то $\varepsilon(2)=\lceil 2 k+1\rceil=$ $=2 k+1$;

- наконец, если $p=8 k+7$, то $\varepsilon(2)=$ $=\left\lceil 2 k+\frac{3}{2}\right\rceil=2 k+2$.

Гипотеза Эйлера. Посмотрим еще раз на предложение 6. Оно утверждает, что 2 является или не является квадратичным вычетом по модулю $p$ в зависимости от 
остатка, который $p$ дает при делении на 8. Вообще говоря, это совершенно удивительно: оказывается, что при фиксированном нечетном $r$ для всех простых чисел вида $8 k+r$ двойка является или не является квадратичным вычетом одновременно!

Кстати, то же самое мы видели и для -1 : является ли -1 вычетом или невычетом по модулю $p$, зависит лишь от остатка, который $p$ дает при делении на 4 .

Эйлер высказал гипотезу, что так будет и для любого числа $a$ : символ Лежандра $\left(\frac{a}{p}\right)$ будет зависеть только от остатка, который $p$ дает при делении на $4 a$.

Сам Эйлер доказать эту гипотезу не смог; это было сделано Гауссом, который установил, как связаны между собой квадратичные вычеты по различным простым модулям. Это утверждение он назвал золотой теоремой (Theorema Aureum), а нам оно сегодня известно как квадратичный закон взаимности. В следующем разделе мы изложим одно из многочисленных доказательств этой теоремы, принадлежащее ученику Гаусса, Эйзенштейну.

Кстати, слово «многочисленные» - отнюдь не преувеличение. Существуют доказательства квадратичного закона взаимности, опирающиеся на самые разные идеи; сам Гаусс придумал около восьми разных доказательств. Немецкий математик Франц Леммермайер на своей странице https: / / www.rzuser.uni-heidelberg.de / hb3/ rchrono.html собрал 246 различных доказательств этой теоремы, последние 15 из которых были опубликованы в течение последнего десятилетия.

Теорема 5 (гипотеза Эйлера). Число $a$ одновременно будет квадратичным вычетом или невычетом для всех простых чисел, входящих в арифметическую прогрессию $4 a n+r$, где $n=0,1,2, \ldots u 0<$ $<r<4 a$.

Замечание. Понятно, что если первый член прогрессии $r$ и ее разность $4 a$ не взаимно просты, то таких простых чисел при $n \geq 1$ не будет (все члены прогрессии будут делиться на их общий делитель). теорема Дирихле утверждает, что если первый член и разность арифметической прогрессии взаимно просты, то она будет содержать бесконечно много простых чисел.

Упражнение 6. Докажите, что количество простых чисел вида: а) $\left.4 k+3 ; \sigma^{*}\right) 4 k+1$ бесконечно.

\section{Квадратичный закон взаимности Гаусса}

Формула для $\varepsilon(\boldsymbol{a})$. В предыдущем разделе мы вычислили $\varepsilon(2)$, найдя число таких «положительных» элементов из $\mathbb{Z} / p \mathbb{Z}$, которые при умножении на 2 переходят в «отрицательные». А именно, эти элементы получаются из целочисленных $x$, удовлетворяющих двойному неравенству $p / 4<x<p / 2$. Напротив, целочисленные решения неравенства $0<x<p / 4$ соответствуют «положительным» элементам, при умножении на 2 переходящим в «положительные».

Это можно представить себе следующим образом. Расположим остатки от 0 до $p-1$ в вершинах правильного $p$-угольника с координатами $(\cos 2 \pi k / p ; \sin 2 \pi k / p)$. Тогда «положительные» остатки будут отвечать вершинам, лежащим в верхней полуплоскости (выше оси $O x$ ), а «отрицательные» - соответственно, ниже $O x$. Умножение элемента на 2 отвечает удвоению соответствующего угла, так что при этом вершина с номером $k$ перейдет в вершину с номером $2 k$. Поэтому в «положительные» элементы при этом отображении перейдут вершины, лежащие в I и III четвертях, а в «отрицательные» - соответственно, во II и IV. Поэтому интересующее нас число «положительных» элементов, переходящих в «отрицательные», есть не что иное, как количество вершин $p$-угольника, лежащих во II четверти.

Ясно, что это рассуждение можно обобщить для произвольного числа $a$, не только для 2. Для этого разобьем нашу плоскость на $2 a$ одинаковых секторов и покрасим их поочередно в белый и серый цвета, как показано на рисунке 1. Вершины правильного $p$-угольника, попавшие в белые сектора, будут отвечать ненулевым элементам из $\mathbb{Z} / p \mathbb{Z}$, переходящим при умно- 


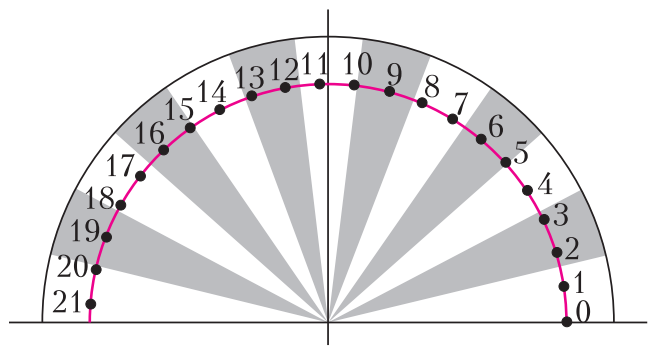

Рис. 1. "Положительные» остатки по модулю $p=43$ (от 1 до 21), попавшие в серые сектора, переходят при умножении на а $=13$ в «отрицательные»

жении на $a$ в «положительные» элементы; те же вершины, которые попали в серые сектора, будут при умножении на $a$ переходить в «отрицательные». На рисунке 1 показан пример, в котором $p=43$ (т.е. «положительными» являются остатки от 1 до 21), а $a=13$.

Отметим, что в силу простоты $p$ (вернее, взаимной простоты $p$ и $a$ ) на границу секторов попадет единственная вершина $p$-угольника - а именно, та, что отвечает элементу $0 \in \mathbb{Z} / p \mathbb{Z}$.

Итак, количество $\varepsilon(a)$ таких «положительных» элементов $\mathbb{Z} / p \mathbb{Z}$, которые при умножении на $a$ переходят в «отрицательные», равняется количеству вершин $p$-угольника, лежащих выше оси абсцисс и при этом попадающих в серые сектора. Всего таких секторов будет $\left\lfloor\frac{a}{2}\right\rfloor$ (т.е. число $\frac{a}{2}$, округленное до ближайшего целого вниз). Ясно, что соответствующие вершины при этом будут иметь номера $n$, удовлетворяющие одному из следующих неравенств:

$$
\begin{gathered}
p / 2<a n<p \\
3 p / 2<a n<2 p \\
5 p / 2<a n<3 p
\end{gathered}
$$

Тем самым, количество «положительных» элементов, переходящих при умножении на $a$ в «отрицательные», есть в точности количество таких $n$, где $1 \leq n \leq \frac{p-1}{2}$ и $\left\lfloor\frac{2 a n}{p}\right\rfloor$ нечетно.
Это можно сформулировать в виде следующей леммы.

Лемма 1 (Эйзенштейн). Имеет место равенство

$$
\left(\frac{a}{p}\right)=(-1) \sum_{n=1}^{(p-1) / 2}\left\lfloor\frac{2 a n}{p}\right\rfloor .
$$

Доказательство. Предыдущее обсуждение показывает, что $\left(\frac{a}{p}\right)$ равно 1 или -1 в зависимости от того, является ли количество нечетных чисел среди чисел вида $\left\lfloor\frac{2 a n}{p}\right\rfloor$ четным или нечетным. Теперь просуммируем все числа такого вида и воспользуемся простым наблюдением: сумма целых чисел нечетна тогда и только тогда, когда в нее входит нечетное число нечетных слагаемых.

Квадратичные вычеты и целые точки. В этом разделе мы установим взаимосвязь между квадратичными вычетами по различным модулям. Для этого зададимся различными простыми нечетными числами $p$ и $q$.

Положим в предыдущей формуле $a=q$ и интерпретируем сумму в ее правой части геометрически. Нарисуем на координатной плоскости прямоугольник высоты $q$ и ширины $p$ с вершиной в начале координат, лежащий в первой четверти (рис.2). Коор-

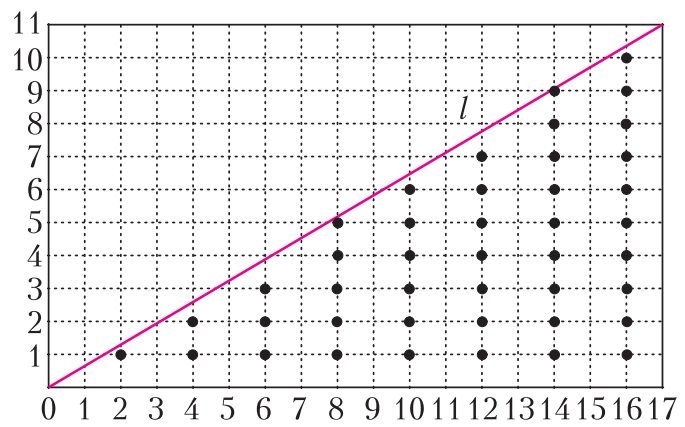

Рис. 2. Здесь $p=17, q=11$. Отмечены все точки с четными абсциссами, лежащие ниже прямой I

динаты его точек $(x ; y)$ будут удовлетворять неравенствам $0<x<p, 0<y<q$. Далее проведем в этом прямоугольнике диагональ $l$ из левого нижнего в правый 
верхний угол; она будет иметь уравнение $y=q x / p$.

Рассмотрим какое-нибудь целое число $n$, где $1 \leq n \leq(p-1) / 2$. Возьмем все точки в прямоугольнике с абсциссой $2 n$ и целой ординатой, лежащие ниже прямой $l$. Их ордината должна удовлетворять условию $y<\frac{2 n q}{p}$. Таким образом, число этих точек равно $\left\lfloor\frac{2 n q}{p}\right\rfloor$. Суммируя по $n$, получаем, что четность $\varepsilon(q)$ равна четности количества точек в прямоугольнике, которые лежат ниже прямой $l$ и имеют четные абсциссы.

Оказывается, что существует еще более удобная интерпретация $\varepsilon(q)$ :

Лемма 2. Четность числа $\varepsilon(q)$ совпадает с четностью числа челых точек в треугольнике, заданном неравенствами $0<x<p / 2, \quad 0<y<q / 2, \quad y<q x / p \quad$ (на рисунке 3 этот треугольник выделен цветом).

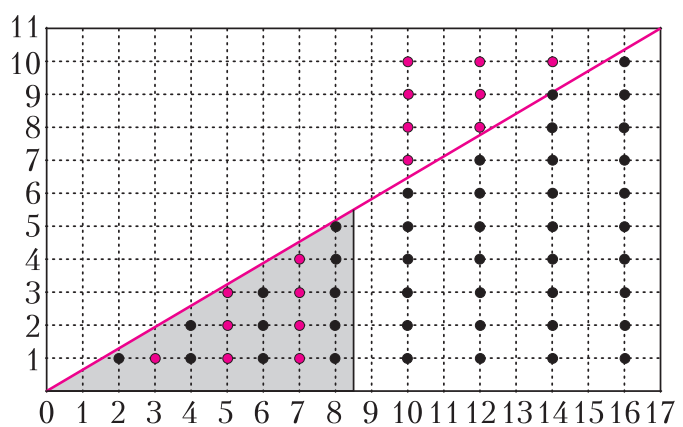

Рис. 3. Четность $\varepsilon(q)$ равна четности числа точек в выделенном треугольнике

Доказательство. Рассмотрим точки, отмеченные на рисунке 2, и добавим к ним еще некоторое количество точек: все точки в выделенном треугольнике, которые еще не отмечены, и центрально-симметричные им относительно центра прямоугольника. На рисунке 3 эти точки отмечены красным. При этом точке с координатами $(x ; y)$ будет соответствовать точка с координатами $(p-x ; q-y)$ - т.е. точке, лежащей ниже прямой $l$, будет отвечать точка выше этой прямой, а точке с нечетной абсциссой будет соответствовать точка, абсцисса ко- торой четна. В результате у нас будут отмечены:

• во-первых, все целые точки в выделенном треугольнике;

- во-вторых, все точки с четными абсциссами, лежащие правее прямой $x=p / 2$. В каждом таком столбце будет $q-1$ точка, т.е. четное число, а значит, общее число таких точек также будет четным.

Значит, четность $\varepsilon(q)$ равна четности числа целых точек в выделенном треугольнике, что и требовалось.

Отсюда немедленно получается квадратичный закон взаимности Гаусса:

Теорема 6 (квадратичный закон взаимности). Для различных нечетных простых чисел имеет место равенство

$$
\left(\frac{p}{q}\right) \cdot\left(\frac{q}{p}\right)=(-1)^{\frac{p-1}{2} \cdot \frac{q-1}{2}} .
$$

Доказательство. В предыдущих обозначениях рассмотрим прямоугольник $0<x<p / 2,0<y<q / 2$. Его делит пополам прямая $l$. Как показывает предыдущее обсуждение, число целых точек под ней будет четным или нечетным в зависимости от знака $\left(\frac{q}{p}\right)$. С другой стороны, четность числа точек над нею определяется знаком $\left(\frac{p}{q}\right)$. Но всего в прямоугольнике содержится $\frac{p-1}{2} \cdot \frac{q-1}{2}$ точек, что и дает нам требуемое утверждение.

На практике квадратичный закон взаимности удобно использовать в следующей форме:

Следствие 4. Пусть $p, q$ - различные нечетные простые числа. Символь Лежандра $\left(\frac{p}{q}\right)$ u $\left(\frac{q}{p}\right)$ равны, если хотя бы одно из чисел $p, q$ имеет вид $4 k+1$, и различны, если они оба имеют вид $4 k+3$.

Из квадратичного закона взаимности легко вывести гипотезу Эйлера (теорема 5).

Доказательство гипотезы Эйлера. Пусть $a$ - такое число, что $p$ и $4 a n+p$ одновре- 
менно являются простыми. Докажем, что

$$
\left(\frac{a}{p}\right)=\left(\frac{a}{4 a n+p}\right) .
$$

Поскольку символ Лежандра мультипликативен, можно считать, что число $a$ тоже является простым (почему?). Если $a=2$, то это утверждение нам уже известно: это предложение 6 . Если же нет, то $a$ нечетно, и можно применить к обеим частям требуемого равенства квадратичный закон взаимности:

$$
\begin{gathered}
\left(\frac{a}{p}\right)=(-1)^{\frac{a-1}{2} \cdot \frac{p-1}{2}}\left(\frac{p}{a}\right) ; \\
\left(\frac{a}{4 a n+p}\right)=(-1)^{\frac{a-1}{2}} \cdot \frac{4 a n+p-1}{2}\left(\frac{4 a n+p}{a}\right)= \\
=(-1)^{\frac{a-1}{2}} \cdot\left(\frac{p-1}{2}+2 a n\right)\left(\frac{p}{a}\right) .
\end{gathered}
$$

Ясно, что эти величины равны. Гипотеза Эйлера доказана.

Рекомендуем читателю, который хочет узнать больше об истории гипотезы Эйлера и квадратичного закона взаимности, прочесть главу о Гауссе в замечательной книге С.Г.Гиндикина «Рассказы о физиках и математиках». В ней, в частности, изложено одно из первых доказательств квадратичного закона, полученное самим Гауссом.
Пример использования квадратичного закона взаимности. В качестве примера выясним с помощью квадратичного закона взаимности, разрешимо ли сравнение

$$
x^{2} \equiv 57(\bmod 179) \text {. }
$$

Для этого нам нужно вычислить $\left(\frac{57}{179}\right)=\left(\frac{3}{179}\right) \cdot\left(\frac{19}{179}\right)$. Вычислим отдельно каждый из этих символов Лежандра.

Оба числа 3 и 179 дают при делении на 4 остаток 3. Поэтому

$$
\left(\frac{3}{179}\right)=-\left(\frac{179}{3}\right)=-\left(\frac{-1}{3}\right)=1,
$$

так как -1 является квадратичным невычетом по модулю 3. Значит, 3 - квадратичный вычет по модулю 179.

Далее, по той же причине (19 тоже имеет вид $4 k+3)$ получаем, что $\left(\frac{19}{179}\right)=-\left(\frac{179}{9}\right)=$ $=-\left(\frac{8}{19}\right)=-\left(\frac{2}{19}\right)$ (поскольку $8=2 \cdot 4$, а 4 - полный квадрат). Но число 19 имеет вид $8 k+3$, поэтому 2 является квадратичным невычетом по модулю 19. Стало быть, $\left(\frac{19}{179}\right)=1$, откуда $\left(\frac{57}{179}\right)=1$, и сравнение $x^{2} \equiv 57(\bmod 179)$ разрешимо.

\section{Прозрачное и непрозрачное}

\section{Л.АШКИНАЗИ}

А РИСУНКЕ 5 ПРЕДСТАВЛЕНЫ ФОТОграфии двух образцов - из монокристалла $\mathrm{CaF}_{2}$ и нанокерамики (поликристала) $\mathrm{CaF}_{2}: \mathrm{Ce}^{3+}$. Оба образца - абсолютно прозрачные!

Теперь обсудим преломление. У газов коэффициенты преломления мало отличаются от единицы, и они вообще мало кого волнуют - кроме астрономов. Откройте зимой окно и посмотрите, как елозит пей-

Окончание. Начало - в «Кванте» №8.

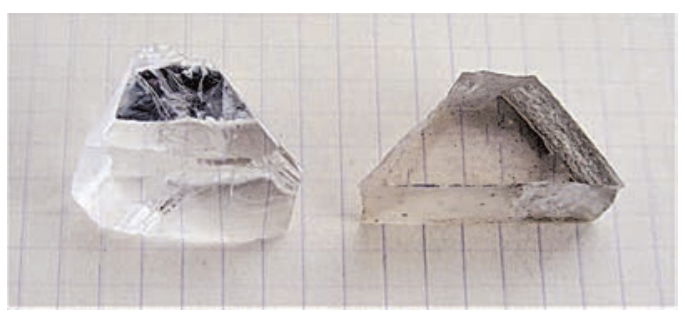

Pис. 5

заж, или просто посмотрите вверх, вспомнив не знаю кем сказанное «человек отличается от животного тем, что иногда под- 